Article

\title{
The Correlation of Surfactant Concentrations on the Properties of Mesoporous Bioactive Glass
}

\author{
Shao-Ju Shih *, Yu-Chien Lin, Leon Valentino Posma Panjaitan and Dyka Rahayu Meyla Sari \\ Received: 22 October 2015; Accepted: 12 January 2016; Published: 19 January 2016 \\ Academic Editor: Gigliola Lusvardi \\ Department of Materials Science and Engineering, National Taiwan University of Science and Technology, 43, \\ Section 4 Keelung Road, Taipei 10607, Taiwan; M10304306@mail.ntust.edu.tw (Y.C.L.); \\ M10004806@mail.ntust.edu.tw (L.V.P.P.); 10204804@mail.ntust.edu.tw (D.R.M.S.) \\ * Correspondence: shao-ju.shih@mail.ntust.edu.tw; Tel.: +886-2-22730-3716; Fax: +886-2-22737-6544
}

\begin{abstract}
Bioactive glass (BG), a potential biomaterial, has received increasing attention since the discovery of its superior bioactivity. One of the main research objectives is to improve the bioactive property of BGs; therefore, surfactant-derived mesoporous bioactive glasses (MBGs) were developed to provide a high specific surface area for achieving higher bioactivity. In this study, various concentrations of typical triblock F127 surfactant were used to manipulate the morphology, specific surface area, and bioactivity of MBG particles. Two typical morphologies of smooth (Type I) and wrinkled (Type II) spheres were observed, and the population of Type II particles increased with an increase in the surfactant concentration. A direct correlation between specific surface area and bioactivity was observed by comparing the data obtained using the nitrogen adsorption-desorption method and in vitro bioactive tests. Furthermore, the optimal surfactant concentration corresponding to the highest bioactivity revealed that the surfactant aggregated to form Type II particles when the surface concentration was higher than the critical micelle concentration, and the high population of Type II particles may reduce the specific surface area because of the loss of bioactivity. Moreover, the formation mechanism of SP-derived MBG particles is discussed.
\end{abstract}

Keywords: mesoporous bioactive glass; morphology; surface area; spray pyrolysis; formation mechanism

\section{Introduction}

$\mathrm{SiO}_{2}, \mathrm{CaO}$, and $\mathrm{P}_{2} \mathrm{O}_{5}$-based bioactive glasses (BGs) have been used as bioactive materials in drug carriers [1,2], dental sealing, and [3] bone implants [4,5] because of their superior bioactivity, which was first proposed by Hench et al. in 1971 [6]. The bioactivity of a material is its ability to form hydroxyl apatite (HA) after it is embedded in the human body $[7,8]$ or immersed in a simulated body fluid (SBF) [9]. HA, the main inorganic bone component, provides the specific biological response of bonding at the tissue-BG interface [10]. Because bioactivity is crucial, improving the bioactive behavior of BGs has attracted considerable attention.

For improving bioactive behaviors, a suitable chemical composition and high specific surface area of BGs are essential, and two methods are available for achieving this goal. First, the basis of the bone-bonding property is the chemical reaction of BGs in body fluids. This reaction involves five steps: (i) the formation of silanol $(\mathrm{SiOH})$; (ii) the loss of soluble silica and formation of $\mathrm{SiOH}$; (iii) polycondensation of $\mathrm{SiOH}$; (iv) the formation of an amorphous calcium phosphate layer; and (v) the crystallization of the HA layer [11]. More details can be found in the study by Hench et al. [11,12]. They claimed that the kinetics of these reaction stages depend on the BG composition [11]. For example, the first and second stages are associated with the $\mathrm{SiOH}$ formation, which is essential for the growth of $\mathrm{HA}$ [11]. A previous study reported that non-bridging oxygen (NBO) groups exchange $\mathrm{H}^{+}$or $\mathrm{H}_{3} \mathrm{O}$ 
from the surrounding SBFs to increase the $\mathrm{SiOH}$ formation [11]. Consequently, in BGs, Ca acts as a modifier to reduce the degree of connectivity in the glassy structure for increasing the population of NBO groups. Therefore, a higher Ca concentration (e.g., $45 \mathrm{~S} 5$ glass [12]) provides more NBO groups, leading to a high dissolution rate and high bioactivity.

The other approach for achieving a high bioactivity is to increase the HA formation rate by maximizing the specific surface area. For example, Mačković et al. reported that the 45S5 BG nanoparticles exhibit a higher surface area and a faster HA formation (immersion in an SBF for 1 day) than micron-sized particles do (immersion in an SBF for 3 days) [13]. Furthermore, Yan et al. used surfactants to prepare a well-ordered mesoporous bioactive glass (MBG) with a high specific surface area [14], and, consequently, numerous studies have used the mesoporous structure to obtain a high bioactivity (HA formation after immersion in an SBF 4-8 h) $[15,16]$. The mesoporous structure has become a requirement for obtaining BG materials.

Because the mesoporous structure is crucial, two methods, sol-gel [14-16] and spray pyrolysis (SP) [17], have been used to fabricate MBGs. Compared to sol-gel, SP is faster and more continuous [18]. Although SP-derived MBGs have the potential for mass production, inhomogeneous particles (smooth and wrinkled spheres [17]) have been observed in SP-derived MBGs. This inhomogeneity may result in the loss of specific surface area, and, based on our research, studies that describe the relationship between morphology and specific surface area for SP-derived MBG particles are rare. For a mesoporous structure, surfactants play a crucial role to form micelles as a template for conducting pore formation [19]. When the surfactant concentration in an aqueous solution is low, surfactants are located as separate molecules in the water and are unable to form micelle channels. When the surfactant concentration increases and reaches a critical value, the surfactant molecules come together to form micelles, and this concentration is called the critical micelle concentration (CMC) [19]. The uses of surfactant for the MBGs, especially when the concentration is higher than the CMC, were reported by Yan et al. [20] and Soulie et al. [21]. However, increasing the concentration further causes the surfactant molecules to aggregate and produce elongated tubes and stacked lamellae at the second and third CMCs, respectively [22,23]. In addition, Myers et al. predicted that the morphologies of the surfactant at high concentrations are spherical, rod-shaped, and have a flexible bilayer structure, planar extended bilayers, and reversed micelles [24]. These morphologies may deteriorate the compacted mesoporous structure and reduce the specific surface area. Therefore, the surfactant concentration is related to the formation of mesoporous structures and affects the specific surface area and bioactivity of MBG particles.

In this study, the common triblock surfactant, F127 (Pluronic F-127, $\mathrm{EO}_{106} \mathrm{PO}_{70} \mathrm{EO}_{106}$, where EO is polyethylene oxide and $\mathrm{PO}$ is polypropylene oxide (Sigma-Aldrich, Ludwigshafen, Germany) was used to produce the mesoporous structure of BG powder, and various surfactant concentration-treated MBG powders were synthesized and characterized to examine the relationship among surfactant concentration, specific surface area, and bioactivity. First, X-ray diffraction (XRD) was used to characterize the phase compositions of these MBG powders. Second, scanning electron microscopy (SEM) and transmission electron microscopy (TEM) were used to observe their surface structures and geometries. In addition, particle size distributions and pore sizes were acquired using a couple of SEM and TEM images, respectively. Third, the specific surface areas of the powders were determined using the nitrogen adsorption-desorption method (Brunauer-Emmett-Teller (BET) method) and were compared with particle morphologies. Finally, for in vitro bioactive tests, the Fourier transform infrared reflection (FTIR) spectrophotometer (Varian Inc., Palo Alto, CA, USA) was used to characterize the powders after they were soaked in an SBF for 1 day, and the intensity of $\mathrm{P}-\mathrm{O}$ bonding of the various MBG powders were acquired to determine their bioactivity [7]. 


\section{Results}

\subsection{Phase Composition}

Figure 1 shows the phase composition of four MBG powders prepared using distinct F127 (surfactant) concentrations of 14, 31, 44, and $53 \mathrm{wt} \%$. Only a broad band between $2 \theta$ angles of $20.0^{\circ}$ and $37.0^{\circ}$ was detected in each XRD spectrum, indicating that all MBG powders were amorphous and that surfactant concentration did not affect the phase composition of MBG powders.

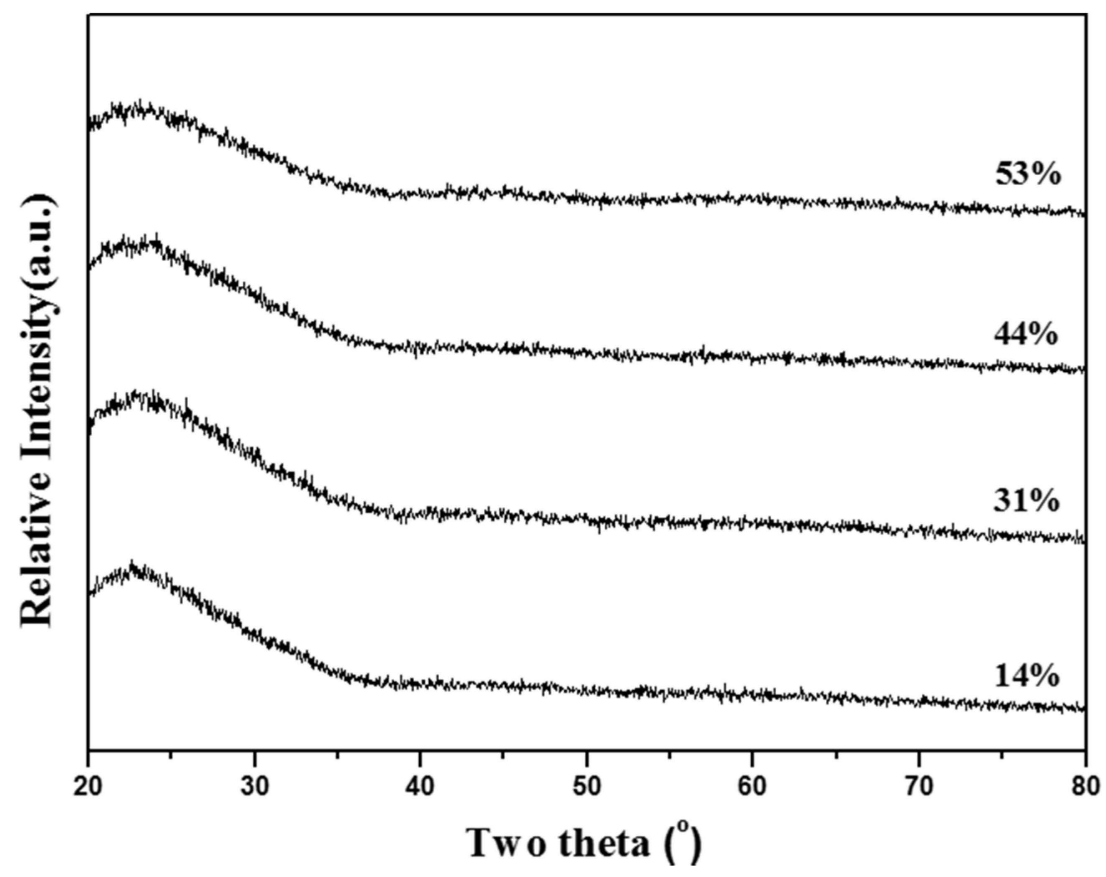

Figure 1. XRD patterns of MBG particles prepared using various surfactant concentrations.

\subsection{Morphology}

For a detailed morphology, the surface structure and geometry of the MBG powders were characterized using SEM and TEM, respectively. Figure 2 shows the SEM micrographs of four MBG powders prepared using different surfactant concentrations. A smooth spherical surface structure was observed for $14 \mathrm{wt} \%$ F127-treated MBG particles (Figure 2a). In addition, the same smooth spherical structure was observed for 31 wt \% F127-treated MBG particles (Figure 2b). However, for 44 and 53 wt \% F127-treated MBG particles, the SEM micrographs reveal two surface structures, smooth and wrinkled spheres, of MBG particles (Figure 2c,d). Furthermore, the particle geometries of MBG powders were investigated using TEM (Figure 3). Figure 3a shows bright and dark regions within a particle, and these bright and dark regions corresponded to pores and walls [25], thus proving the mesopore formation within the $14 \mathrm{wt} \%$ F127-treated MBG particles. In addition, the same geometry was observed for the $31 \mathrm{wt} \%$ F127-treated MBG particles (Figure 3b). However, for 44 and 53 wt \% F127-treated MBG particles, both a mesoporous structure and an irregular surface were observed (Figure 3c,d). Furthermore, from the TEM images, the average values and standard deviations of pore size for 14, 31, 44, and 53 wt \% F127 were $6.9 \pm 1.3,7.4 \pm 1.5,7.1 \pm 1.4$, and $5.5 \pm 1.3 \mathrm{~nm}$, respectively. By combining the SEM and TEM micrographs, two morphologies, smooth mesoporous (Type I) and wrinkled mesoporous (Type II), were observed. This result suggests that MBG particles treated with lower F127 concentrations (14 wt \% and 31 wt \% F127-treated MBG particles) exhibited only Type I morphology, whereas those treated with higher F127 concentrations (44 and 53 wt \% F127-treated MBG particles) 
exhibited both Type I and Type II morphologies. This implies that the MBG morphology is a function of F127 concentration. In summary, particle morphologies were constructed using surface structure and geometry, which were observed using SEM and TEM.
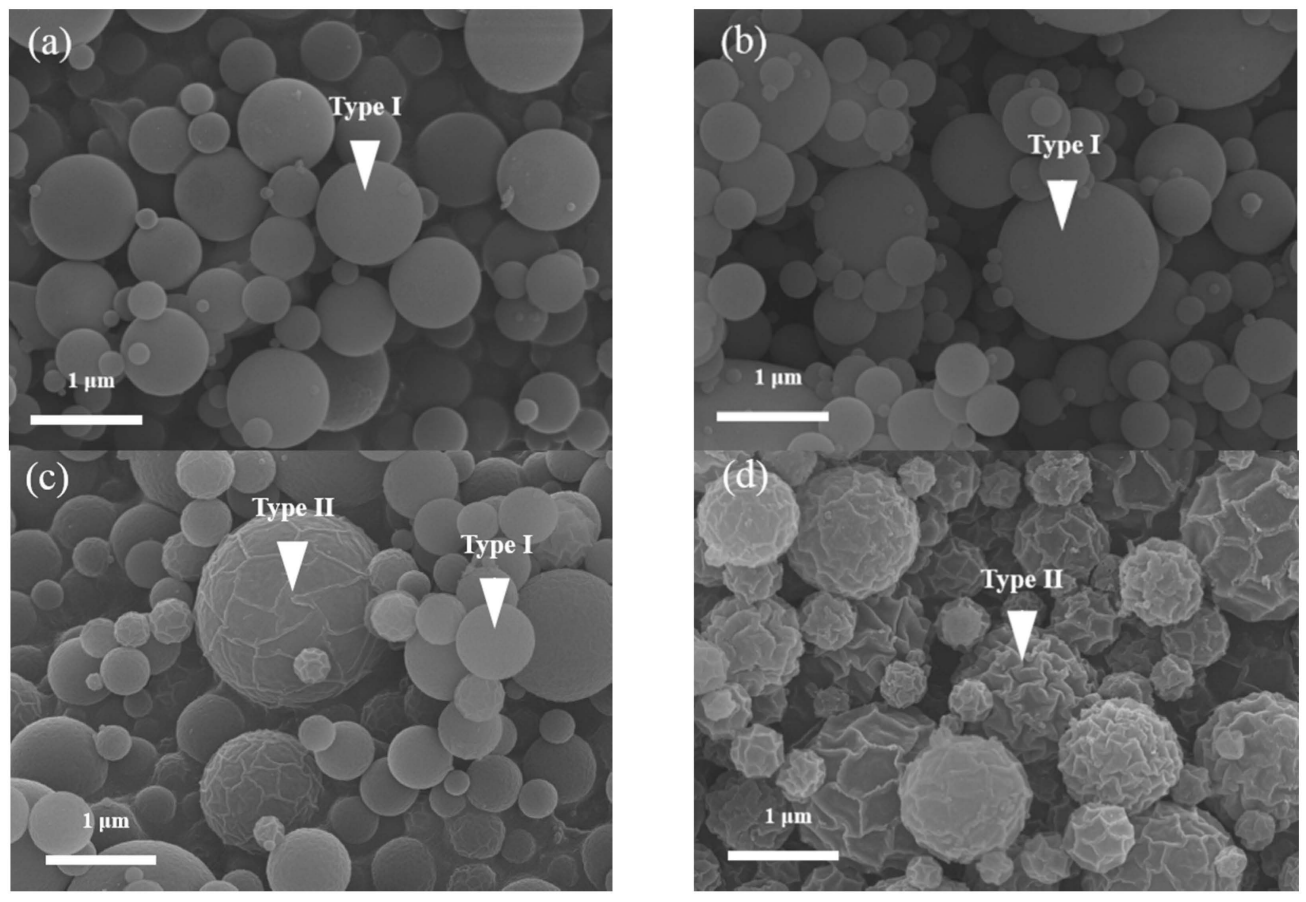

Figure 2. SEM images of MBG particles prepared using various surfactant concentrations of (a) $14 \mathrm{wt} \%$; (b) $31 \mathrm{wt} \%$; (c) $44 \mathrm{wt} \%$; and (d) $53 \mathrm{wt} \%$.
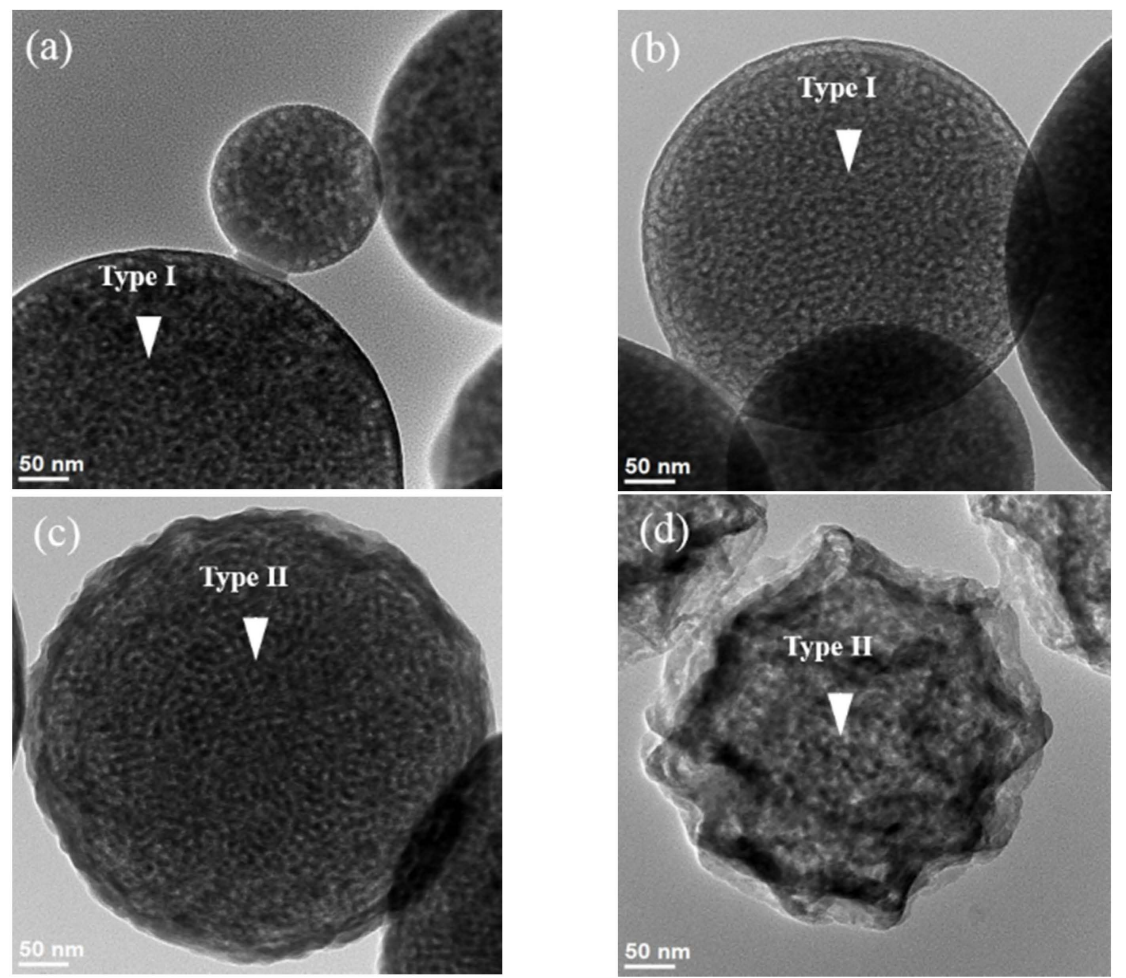

Figure 3. TEM images of MBG particles prepared using various surfactant concentrations of (a) $14 \mathrm{wt} \%$; (b) $31 \mathrm{wt} \%$; (c) $44 \mathrm{wt} \%$; and (d) $53 \mathrm{wt} \%$. 


\subsection{Particle Size Distribution and Specific Surface Area}

The particle size distributions of various F127-treated MBG powders are shown in Figure 4. The average particle sizes and standard deviations of the $14 \mathrm{wt} \%, 31 \mathrm{wt} \%, 44 \mathrm{wt} \%$, and $53 \mathrm{wt} \%$ F127-treated MBG powders were $579 \pm 275,878 \pm 675,942 \pm 474$, and $1119 \pm 624 \mathrm{~nm}$, respectively. This result revealed that the particle size increased with an increase in the surfactant concentration. The BET measurements showed that the specific surface areas of the $14 \mathrm{wt} \%, 31 \mathrm{wt} \%, 44 \mathrm{wt} \%$, and $53 \mathrm{wt} \%$ F127-treated MBG powders were $104.2 \pm 19.8,258.3 \pm 21.1,338.9 \pm 12.3$, and $296.6 \pm 12.6 \mathrm{~m}^{2} / \mathrm{g}$, respectively. The BET data showed that the specific surface area had the maximum value of $338.9 \mathrm{~m}^{2} / \mathrm{g}$ when the F127 concentration was $44 \mathrm{wt} \%$. However, when the surfactant concentration was increased to $53 \mathrm{wt} \%$, the specific surface area decreased to $296.6 \mathrm{~m}^{2} / \mathrm{g}$.

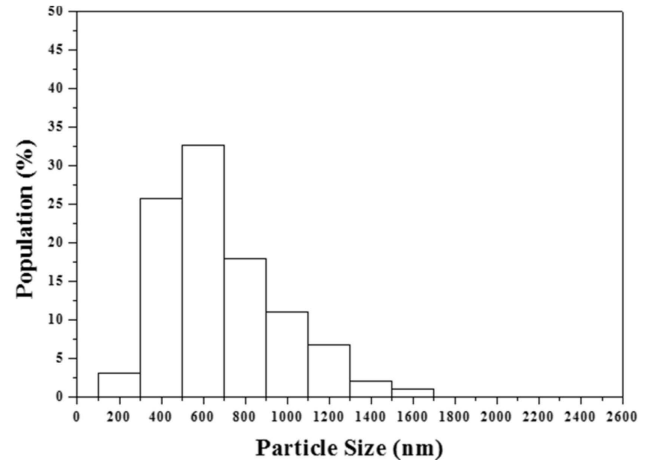

(a)

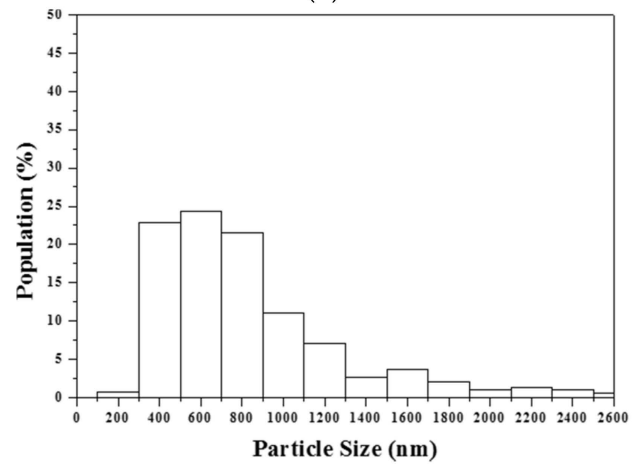

(c)

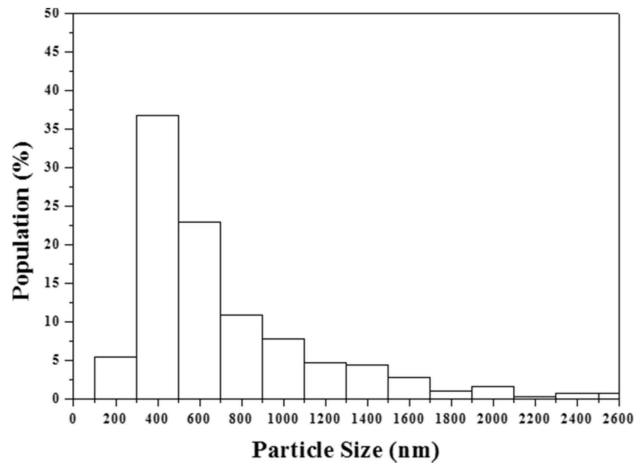

(b)

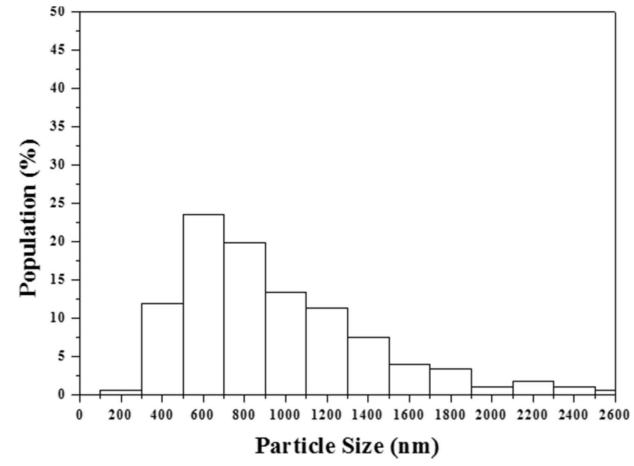

(d)

Figure 4. Particle size distributions of MBG particles prepared using various surfactant concentrations of (a) $14 \mathrm{wt} \%$; (b) $31 \mathrm{wt} \%$; (c) $44 \mathrm{wt} \%$; and (d) $53 \mathrm{wt} \%$.

\subsection{In Vitro Bioactive Test}

The characterization of the HA crystallinity of SBF-immersed BGs is commonly used to determine the corresponding bioactivities [11]. Figure 5 shows the FTIR patterns of $14 \mathrm{wt} \%, 31 \mathrm{wt} \%, 44 \mathrm{wt} \%$, and $53 \mathrm{wt} \%$ F127-treated MBG powders before and after immersion in an SBF for 1 day. The peaks at 1095 and $482 \mathrm{~cm}^{-1}$ are assigned as a Si-O-Si stretching vibration and a Si-O-Si bending mode, respectively [26,27]. The peaks at 598 and $566 \mathrm{~cm}^{-1}$ are assigned as $\mathrm{P}-\mathrm{O}$ bending vibrations in $\mathrm{PO}_{4}$ tetrahedra [28]. The bioactivity is determined by obtaining a ratio of peak intensities from the FTIR patterns. $\mathrm{I}_{1}$ refers to the intensity of $\mathrm{P}-\mathrm{O}$ bending vibration around $566 \mathrm{~cm}^{-1}$, whereas $\mathrm{I}_{2}$ refers to the intensity of Si-O-Si bending vibration at $482 \mathrm{~cm}^{-1}$. The $\mathrm{I}_{1} / \mathrm{I}_{2}$ value corresponds the amount of HA: the higher the $I_{1} / I_{2}$ the larger amount of HA. Note that all MBG powders exhibited an amorphous structure before immersion in an SBF (Figure 1). After immersion in an SBF for 1 day, bioactivity was directly related to the HA formation rates after immersion in an SBF and in turn to the HA amount after the same SBF immersion time. The $\mathrm{I}_{1} / \mathrm{I}_{2}$ values of $14,31,44$, and 53 wt \% F127-treated MBG powders 
are $0.18,0.24,0.37$ and 0.28 , respectively; in other words, the $\mathrm{I}_{1} / \mathrm{I}_{2}$ value and the corresponding bioactivity followed the order $44 \%>53 \%>31 \%>14 \%$.

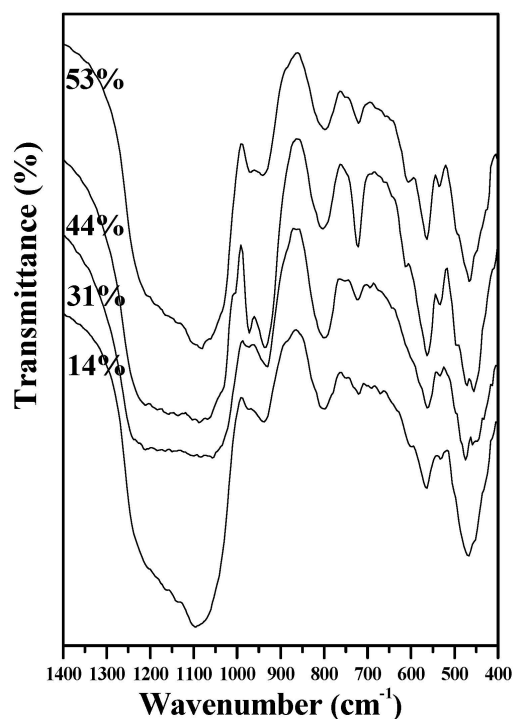

Figure 5. FTIR patterns of various surfactant concentration treated MBG particles immersed in SBF for 1 day.

\section{Discussion}

In SP, the problem of inhomogeneity is frequently observed during the preparation of particles, including elemental particles (e.g., $\mathrm{SrTiO}_{3}$ [29] and $\mathrm{CaMgSi}_{2} \mathrm{O}_{6}$ [30]) because different precursors have different precipitation rates. In a previous study, we used TEOS, CN, and TEP as precursors $(\mathrm{Si}, \mathrm{Ca}$, and $\mathrm{P}$ precursors, respectively) to prepare SP-derived $\mathrm{BG}$ at a high calcination temperature of $700{ }^{\circ} \mathrm{C}$, and the nanoparticles of amorphous $\mathrm{SiO}_{2}$ and the submicron particles of wollastonite-based glass were observed using TEM and X-ray energy-dispersive spectroscopy [31]. TEOS is insoluble with water because of its hydrolysis behavior, whereas $\mathrm{CN}$ and TEP are soluble in water, and the different precipitation behaviors of TEOS, CN, and TEP led to this inhomogeneity. In this study, TEOS, CN, and TEP were used to prepare MBG particles. However, no segregation or any second phase was detected in the XRD data (Figure 1) for any of the MBG particles (with treatments with various surfactant concentrations). This result implies that the hydrophobic PO groups and hydrophilic EO groups of the F127 surfactant attracted the insoluble TEOS and soluble CN and TEP during heating to avoid the problem of phase separation.

Two morphologies, smooth (Type I) and wrinkled (Type II) spheres, were observed. The Type I particles were observed frequently and were described in-depth in our previous studies $[17,25]$. A possible explanation for the formation of Type II particles is that the concentration of the partial droplets of the surfactant exceeded the $\mathrm{CMC}$, causing the particles to aggregate to produce spherical micelles, elongated tubes, and stacked lamellae at the first, second, and third CMCs, respectively [22]. After SP, these tubes and stacked lamellae burned out, and the wrinkled mesoporous spheres were obtained. According to statistical analysis, the population of Type II particles for the $14 \mathrm{wt} \%, 31 \mathrm{wt} \%$, $44 \mathrm{wt} \%$, and $53 \mathrm{wt} \% \mathrm{~F} 127$-treated MBG powders were $0.0 \%, 0.0 \%, 44.6 \%$, and $96.1 \%$ (the population of Type I particles were $100.0 \%, 100.0 \%, 55.4 \%$, and $3.9 \%$ ), respectively. This statistical result showed that (i) the CMC of F127 in this study was between 33 and $44 \mathrm{wt} \%$ and (ii) the population (\%) of Type II particles increased with an increase in the surfactant concentration. In summary, the population of wrinkled spherical particles was closely related to the surfactant concentration.

The statistical analysis suggested a direct relation between particle size and surfactant concentration; the higher the surfactant concentration, the larger the particle size. It is well known 
that the particle size is a function of precursor, solid content in the precursor solution, and ultrasonic frequency [32]. In this study, the solid content in the precursor solution and ultrasonic frequency were fixed. The result suggested that the precursor solution of a high surfactant concentration provides more porosity to form larger particles than a lower surfactant concentration, which is supported by TEM results (Figure 3). In addition, there was no considerable correlation between particle size and particle morphology; the average particle sizes and their standard deviations for Type I and II particles were $1.12 \pm 0.66$ and $0.85 \pm 0.66 \mu \mathrm{m}$, respectively. This indicates the distribution of elongated tubes and stacked lamellae was homogenous.

Furthermore, a detailed examination showed that all MBG powders exhibited a normal size distribution (submicron size) and not the bimodal distribution (nano and submicron size). A normal size distribution implied that the particle formation mechanism involved only "one-particle-per-drop" and not "gas-to-particle conversion" [33]. Furthermore, for the case of spray pyrolyzed BG (without addition of surfactant) [26], the effects of phase separation on loss of bioactivity were investigated. This phase-separated BG powder contains the submicron glass ceramic particles, with the amorphous phases and the minor phases of monoclinic wollastonite (JCPDS of 43-1460) and triclinic wollastonite (JCPDS 29-0372), and the amorphous $\mathrm{SiO}_{2}$ nanoparticles. These wollastonite phases reduce the amorphous region, which is responsible for bioactivity; therefore, the phase separation deteriorates the bioactivity of BG. So, the formation of amorphous $\mathrm{SiO}_{2}$ nanoparticles inhibits the formation of HA. This result supports that the view that the addition of a surfactant stabilizes the Si precursor and avoids the formation of amorphous $\mathrm{SiO}_{2}$ nanoparticles [31] that cause the gas-to-particle conversion for phase separation.

Although a high specific surface area of MBGs leads to a high bioactivity [7], the data of specific surface area must be compared with those of the bioactivity test to determine the relationship between bioactivity and specific surface area. Figure 6 shows the relationship between HA bioactivity (the $\mathrm{I}_{1} / \mathrm{I}_{2}$ value) and the corresponding specific surface area. The $\mathrm{I}_{1} / \mathrm{I}_{2}$ value (i.e., bioactivity) increased with an increase in the specific surface area. The BET data suggested that the specific surface area of the MBG powders followed the order $44 \%\left(338.9 \mathrm{~m}^{2} / \mathrm{g}\right)>53 \%\left(296.6 \mathrm{~m}^{2} / \mathrm{g}\right)>31 \%\left(258.3 \mathrm{~m}^{2} / \mathrm{g}\right)$ $>14 \%\left(104.2 \mathrm{~m}^{2} / \mathrm{g}\right)$, which is consistent with the bioactivity order $\left.\left(\mathrm{I}_{1} / \mathrm{I}_{2}\right)\right)(44 \%(0.37)>53 \%(0.28)$ $>31 \%(0.24)>14 \%(0.18))$. Therefore, maximizing the specific surface area is critical for bioactivity improvement in SP-derived MBG particles.

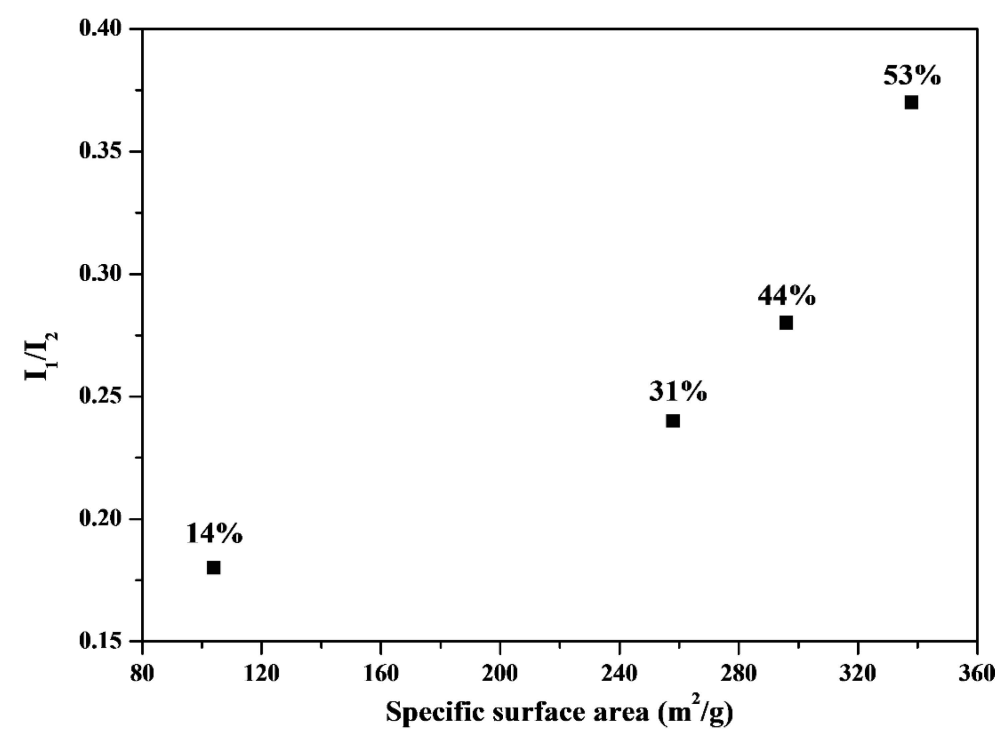

Figure 6. Comparison of $\mathrm{I}_{1} / \mathrm{I}_{2}$ (bioactivity) and surface area for various surfactant concentrations treated MBG. The values of surfactant concentration are given. 
Previous studies have reported that the specific surface area of an MBG is determined by its pore size [5] and particle morphology [17]. First, we considered the case of MBG powders with a similar pore size to minimize the influence of pore size on the specific surface area. For example, $14 \mathrm{wt} \%$, $31 \mathrm{wt} \%$, and $44 \mathrm{wt} \%$ F127-treated MBG powders had a similar pore size of $7 \mathrm{~nm}$, and the specific surface area increased with an increase in the surfactant concentration $\left(104.2,258.3\right.$, and $338.9 \mathrm{~m}^{2} / \mathrm{g}$ for $14 \mathrm{wt} \%, 31 \mathrm{wt} \%$, and $44 \mathrm{wt} \%$, respectively), implying that a MBG powder with a high surfactant concentration provides more micelle tubes, resulting in a high specific surface area (i.e., a high density of the mesopores). Furthermore, in principle, a $53 \mathrm{wt} \%$ F127-treated MBG powder should contain the highest amount of micelle tubes and the largest specific surface area. However, the BET value of the $53 \mathrm{wt} \%$ F127-treated MBG powder was lower than that of the 44 wt \% F127-treated MBG powder. A possible explanation is that in the case of $53 \mathrm{wt} \%$ F127-treated MBG powder, in precursor droplets, the surfactant concentration is higher than its CMC (between $33 \mathrm{wt} \%$ and $44 \mathrm{wt} \%$ ), causing the micelles to aggregate and produce stacked lamellae and then settle on the droplets because of a lower density than water. After sintering, the organic moieties of these lamellae are burned to form the wrinkled structure (Type II). Figure 7 shows the particle morphology as a function of the surfactant concentration. The population of Type II morphology increased from $44.6 \%$ to $96.1 \%$ when the surfactant concentration was increased from $44 \mathrm{wt} \%$ to $53 \mathrm{wt} \%$. This wrinkled structure may be attributable to the loss of the specific surface area because the structure reduced the mesopore density on the MBG surface (Figure 3d).

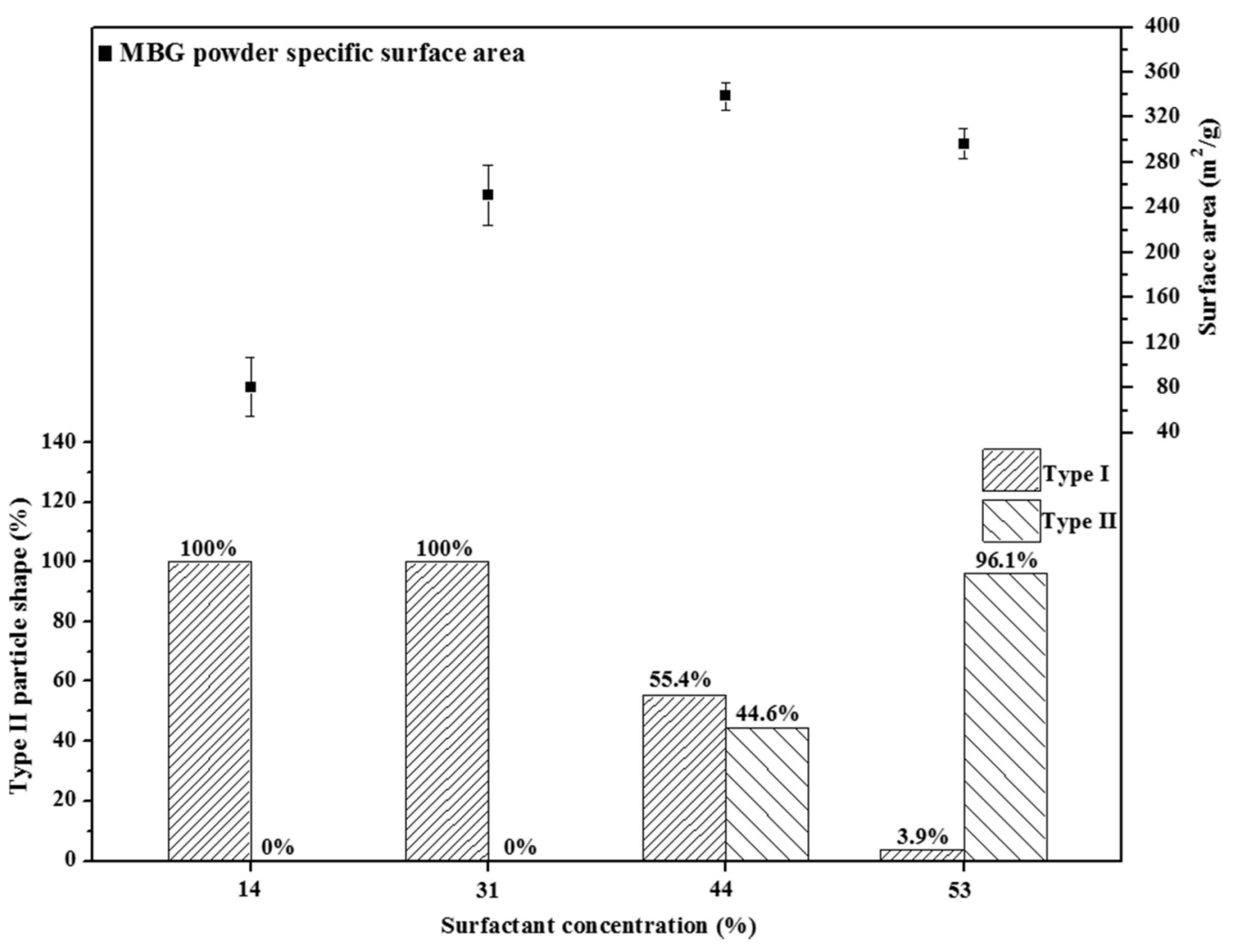

Figure 7. Relationship between particle shape and surface area for various surfactant concentrations treated MBG powders. The percentage values of Type I and II are given.

In summary, a suitable surfactant concentration that provides a high mesopore density and avoids the formation of aggregated surfactant lamellae must be determined for maximizing the specific surface area and in turn obtaining high bioactivity in MBGs. 


\section{Materials and Methods}

\subsection{Synthesis}

The $\mathrm{SiO}_{2}-\mathrm{CaO}-\mathrm{P}_{2} \mathrm{O}_{5}$ MBG powders were prepared from four F127 surfactant concentrations of $14 \mathrm{wt} \%, 31 \mathrm{wt} \%, 44 \mathrm{wt} \%$ and $53 \mathrm{wt} \%$, using SP. Initially, the Si, Ca, and P precursors are $6.70 \mathrm{~g}$ tetraethyl orthosilicate (TEOS, $\mathrm{Si}\left(\mathrm{OC}_{2} \mathrm{H}_{5}\right)_{4}, 99.9 \mathrm{wt} \%$, Showa, Osaka, Japan), $1.40 \mathrm{~g}$ calcium nitrate tetrahydrate $\left(\mathrm{CN}, \mathrm{Ca}\left(\mathrm{NO}_{3}\right)_{2} 4 \mathrm{H}_{2} \mathrm{O}, 98.5 \mathrm{wt} \%\right.$, Showa, Osaka, Japan) and $0.73 \mathrm{~g}$ triethyl phosphate (TEP, $\left(\mathrm{C}_{2} \mathrm{H}_{5}\right)_{3} \mathrm{PO}_{4}, 99 \mathrm{wt} \%$, Alfa Aesar, Haverhill, MA, USA), respectively for the Si:Ca:P molar ratio of 80:15:5. Then, these precursors were mixed with 1.44, 3.97, 6.95, and $9.97 \mathrm{~g}$ F127 surfactants (for surfactant concentrations of $14 \mathrm{wt} \%, 31 \mathrm{wt} \%, 44 \mathrm{wt} \%$ and $53 \mathrm{wt} \%$ ), dissolved in $1.00 \mathrm{~g} 0.5 \mathrm{M} \mathrm{HCl}$ and $60.00 \mathrm{~g}$ ethanol and stirred at room temperature for $24 \mathrm{~h}$ to form precursor solutions. For the SP process, the $10 \mathrm{~mL}$ of precursor solution was mixed with $90 \mathrm{~mL}$ of DI water to disperse into fine droplets using the nebulizer (King Ultrasonics Co., New Taipei, Taiwan) at the frequency of $1.65 \mathrm{MHz}$. Subsequently, the droplets formed particles in a tube furnace (D110, Dengyng, New Taipei, Taiwan), which has three preheating, calcination, and cooling zones of 400,700 , and $500{ }^{\circ} \mathrm{C}$. The surfaces of the particles were then charged by electrons released from tungsten corona wire at high voltage $(16 \mathrm{kV})$. Finally, the negatively charged powders were neutralized and condensed in an earthed stainless steel collector.

\subsection{Characterization}

In order to understand the detailed structures of MBG powders prepared using various F127 concentrations $(14,31,44$ and $53 \mathrm{wt} \%)$, the data of phase composition, surface structure, geometry, and specific surface area were examined using XRD, SEM, TEM and BET, respectively. Firstly, the phase composition was observed using the X-ray diffractometer (D2 Phaser, Billerica, MA, USA) with the operating voltage of $30 \mathrm{kV}$, the operating current of $10 \mathrm{~mA}$, the scan range of $2 \theta$ angle from $20^{\circ}$ to $50^{\circ}$ and the scan rate of $1^{\circ} \mathrm{s}^{-1}$. Secondly, the surface morphology was observed by using field-emission SEM (JSM-6500F, JEOL, Tokyo, Japan), and, for each MBG powder, over 300 MBG particles from a few SEM micrographs have been acquired to obtain the corresponding particle size distribution. Thirdly, field-emission TEM (Tecnai G2 F20, FEI, Hillsboro, OR, USA) was carried out to exam the detailed geometries and pore sizes of MBG; more than 100 pores were used to obtain the average value and standard deviation of pore size. Finally, the specific surface areas of various MBG powders were measured by the Brunauer-Emmett-Teller (BET) method with nitrogen adsorption and desorption isotherms under the temperature of $-196^{\circ} \mathrm{C}$; the powders were degassed at $400{ }^{\circ} \mathrm{C}$ for $3 \mathrm{~h}$ before the BET measurements.

In addition, the test solution of Kokubo's simulated body fluid (SBF) [9] has been previously used for in vitro bioactivity tests. The MBG powders were immersed in SBF at $37^{\circ} \mathrm{C}$ for 1 day. The amount of SBF was adjusted to the specific surface areas of MBG. Solid to liquid ratio of $2 \mathrm{mg}$ to $10 \mathrm{~mL}$ for the case of $14 \mathrm{wt} \%, 2 \mathrm{mg}$ to $25 \mathrm{~mL}$ for the case of $31 \mathrm{wt} \%, 2 \mathrm{mg}$ to $33 \mathrm{~mL}$ for the case of $44 \mathrm{wt} \%$ and $2 \mathrm{mg}$ to $29 \mathrm{~mL}$ for the case of $53 \mathrm{wt} \%$. Then, the immersed powers were dried at $100{ }^{\circ} \mathrm{C}$ for 1 day, and the phase composition of immersed powders was characterized using XRD to examine the bioactivity of MBG, the HA formation ability of MBG in SBF. The quantitative values of bioactivity of MBG powders were determined using the peak area from the largest peak of HA.

\section{Conclusions}

MBG powders treated with various F127 surfactant concentrations (14, 31, 44, and 53 wt \%) were successfully synthesized using SP. The XRD patterns showed that all MBG powders were amorphous and without phase separation. In addition, two morphologies, smooth (Type I) and wrinkled (Type II) spheres, were detected using SEM and TEM. The population of Type II particles is a function of the surfactant concentration because, when the surfactant concentration was higher than its CMC, aggregated micelles were formed, in turn forming wrinkled spherical particles. Furthermore, more surfactants produced more micelles in precursor droplets, and the porosity increased after calcination, 
resulting in a high specific surface area. However, the MBG powder treated with the highest surfactant concentration (53 wt \%) did not exhibit the highest specific surface area because the wrinkled structure reduced the mesopore density. Moreover, in vitro bioactive tests suggested that the order of HA formation rate was consistent with that of the specific surface area. Finally, a suitable surfactant concentration must be determined to obtain the maximum bioactivity of SP-derived MBG powders.

Acknowledgments: The authors acknowledge the financial support from the National Science Council of Taiwan (Grant Numbers of NSC 101-2628-E-011-008-MY2 and NSC 103-2221-E-011-031).

Author Contributions: The study was designed by Shao-Ju Shih. The data collection was performed by Leon Valentino Posma Panjaitan and Dyka Rahayu Meyla Sari. The data analysis was performed by Yu-Chien Lin. The overall planning was directed by Shao-Ju Shih.

Conflicts of Interest: The authors declare no conflict of interest.

\section{References}

1. Vallet-Regí, M.; Balas, F.; Colilla, M.; Manzano, M. Bone-regenerative bioceramic implants with drug and protein controlled delivery capability. Prog. Solid State Chem. 2008, 36, 163-191. [CrossRef]

2. Christie, J.K.; Malik, J.; Tilocca, A. Bioactive glasses as potential radioisotope vectors for in situ cancer therapy: Investigating the structural effects of yttrium. Phys. Chem. Chem. Phys. 2011, 13, 17749-17755. [CrossRef] [PubMed]

3. Chatzistavrou, X.; Tsigkou, O.; Amin, H.D.; Paraskevopoulos, K.M.; Salih, V.; Boccaccini, A.R. Sol-gel based fabrication and characterization of new bioactive glass-ceramic composites for dental applications. J. Eur. Ceram. Soc. 2012, 32, 3051-3061. [CrossRef]

4. Hench, L.L.; Xynos, I.D.; Polak, J.M. Bioactive glasses for in situ tissue regeneration. J. Biomater. Sci.-Polym. Ed. 2004, 15, 543-562. [CrossRef] [PubMed]

5. Shih, C.J.; Chen, H.T.; Huang, L.F.; Lu, P.S.; Chang, H.F.; Chang, I.L. Synthesis and in vitro bioactivity of mesoporous bioactive glass scaffolds. Mater. Sci. Eng. C-Mater. Biol. Appl. 2010, 30, 657-663. [CrossRef]

6. Hench, L.L.; Splinter, R.J.; Allen, W.; Greenlee, T. Bonding mechanisms at the interface of ceramic prosthetic materials. J. Biomed. Mater. Res. 1971, 5, 117-141. [CrossRef]

7. Li, R.; Clark, A.; Hench, L. An investigation of bioactive glass powders by sol-gel processing. J. Appl. Biomater. 1991, 2, 231-239. [CrossRef] [PubMed]

8. Xia, W.; Chang, J. Preparation, in vitro bioactivity and drug release property of well-ordered mesoporous 58s bioactive glass. J. Non-Cryst. Solids 2008, 354, 1338-1341. [CrossRef]

9. Kokubo, T.; Kushitani, H.; Sakka, S.; Kitsugi, T.; Yamamuro, T. Solutions able to reproduce in vivo surface-structure changes in bioactive glass-ceramic a-w3. J. Biomed. Mater. Res. 1990, 24, 721-734. [CrossRef] [PubMed]

10. Cao, W.P.; Hench, L.L. Bioactive materials. Ceram. Int. 1996, 22, 493-507. [CrossRef]

11. Hench, L.L. Bioceramics: From concept to clinic. J. Am. Ceram. Soc. 1991, 74, 1487-1510. [CrossRef]

12. Hench, L.L. Bioactive ceramics. In Bioceramics: Materials Characteristics Versus in vivo Behaviour; Ducheyne, P., Lemons, J., Eds.; Annals of New York Academy of Science: New York, NY, USA, 1988; Volume 53, p. 54.

13. Mačković, M.; Hoppe, A.; Detsch, R.; Mohn, D.; Stark, W.J.; Spiecker, E.; Boccaccini, A.R. Bioactive glass (type 45s5) nanoparticles: In vitro reactivity on nanoscale and biocompatibility. J. Nanopart. Res. 2012, 14, 966. [CrossRef]

14. Yan, X.X.; Deng, H.X.; Huang, X.H.; Lu, G.Q.; Qiao, S.Z.; Zhao, D.Y.; Yu, C.Z. Mesoporous bioactive glasses. I. Synthesis and structural characterization. J. Non-Cryst. Solids 2005, 351, 3209-3217. [CrossRef]

15. Lei, B.; Chen, X.F.; Wang, Y.G.; Zhao, N.R.; Du, C.; Fang, L.M. Synthesis and bioactive properties of macroporous nanoscale $\mathrm{SiO}_{2}-\mathrm{CaO}-\mathrm{P}_{2} \mathrm{O}_{5}$ bioactive glass. J. Non-Cryst. Solids 2009, 355, 2678-2681. [CrossRef]

16. Yan, X.X.; Wei, G.F.; Zhao, L.Z.; Yi, J.; Deng, H.X.; Wang, L.Z.; Lu, G.Q.; Yu, C.Z. Synthesis and in vitro bioactivity of ordered mesostructured bioactive glasses with adjustable pore sizes. Microporous Mesoporous Mater. 2010, 132, 282-289. [CrossRef]

17. Shih, S.J.; Chou, Y.J.; Chien, I.C. One-step synthesis of bioactive glass by spray pyrolysis. J. Nanopart. Res. 2012, 14, 1299. [CrossRef] 
18. Shih, S.J.; Chang, L.Y.S.; Chen, C.Y.; Borisenko, K.B.; Cockayne, D.J.H. Nanoscale yttrium distribution in yttrium-doped ceria powder. J. Nanopart. Res. 2009, 11, 2145-2152. [CrossRef]

19. Chen, C.Y.; Chiang, C.Y.; Shih, S.J.; Tsay, C.Y.; Lin, C.K. High supercapacitive performance of sol-gel zno-doped manganese oxide coatings. Thin Solid Films 2013, 528, 61-66. [CrossRef]

20. Yan, X.; Yu, C.; Zhou, X.; Tang, J.; Zhao, D. Highly ordered mesoporous bioactive glasses with superior in vitro bone-forming bioactivities. Angew. Chem. Int. Ed. 2004, 43, 5980-5984. [CrossRef] [PubMed]

21. Soulié, J.; Lao, J.; Jallot, E.; Nedelec, J.-M. Influence of mesostructuration on the reactivity of bioactive glasses in biological medium: A pixe-rbs study. J. Mater. Chem. 2012, 22, 20680-20688. [CrossRef]

22. Chen, X.; Mao, S.S. Titanium dioxide nanomaterials: Synthesis, properties, modifications, and applications. Chem. Rev. 2007, 107, 2891-2959. [CrossRef] [PubMed]

23. Kunjappu, J.T.; Somasundaran, P.; Sivadasan, K. A unique conformational equilibrium of polyacrylic acid at the solid/liquid interface. Colloids Surfaces A 1995, 97, 101-107. [CrossRef]

24. Myers, D. Surfaces, Interfaces, and Colloids: Principles and Applications; Wiley-VCH: New York, NY, USA, 1999.

25. Shih, S.J.; Chou, Y.J.; Panjaitan, L.V.P. Synthesis and characterization of spray pyrolyzed mesoporous bioactive glass. Ceram. Int. 2013, 39, 8773-8779. [CrossRef]

26. Bell, R.; Dean, P. Atomic vibrations in vitreous silica. Discuss. Faraday Soc. 1970, 50, 55-61. [CrossRef]

27. Gaskell, P. Vibrational spectra of simple silicate glasses. Discuss. Faraday Soc. 1970, 50, 82-93. [CrossRef]

28. Fowler, B. Infrared studies of apatites. I. Vibrational assignments for calcium, strontium, and barium hydroxyapatites utilizing isotopic substitution. Inorg. Chem. 1974, 13, 194-207. [CrossRef]

29. Nonaka, K.; Hayashi, S.; Okada, K.; Otsuka, N.; Yano, T. Characterization and control of phase segregation in the fine particles of batio $_{3}$ and srtio $_{3}$ synthesized by the spray pyrolysis method. J. Mater. Res. 1991, 6, 1750-1756. [CrossRef]

30. Hayashi, S.; Otsuka, N.; Akiyama, K.; Okada, K.; Yano, T. Preparation of diopside fine powders by spray pyrolysis and its sinterability J. Ceram. Soc. Jpn. 1989, 97, 742-746. [CrossRef]

31. Shih, S.J.; Tzeng, W.-L.; Chou, Y.J.; Chen, C.Y.; Chen, Y.J. The influence of phase separation on bioactiivty of spray pyrolyzed bioactive glass. J. Nanosci. Nanotechnol. 2015, 15, 4688-4696. [CrossRef] [PubMed]

32. Song, Y.L.; Tsai, S.C.; Chen, C.Y.; Tseng, T.K.; Tsai, C.S.; Chen, J.W.; Yao, Y.D. Ultrasonic spray pyrolysis for synthesis of spherical zirconia particles. J. Am. Ceram. Soc. 2004, 87, 1864-1871. [CrossRef]

33. Messing, G.L.; Zhang, S.C.; Jayanthi, G.V. Ceramic powder synthesis by spray-pyrolysis. J. Am. Ceram. Soc. 1993, 76, 2707-2726. [CrossRef]

(C) 2016 by the authors; licensee MDPI, Basel, Switzerland. This article is an open access article distributed under the terms and conditions of the Creative Commons by Attribution (CC-BY) license (http:/ / creativecommons.org/licenses/by/4.0/). 